\title{
BAIXA EFICÁCIA DA ASSOCIAÇÃO OXAMNIQUINE E PRAZIQUANTEL NA CURA DA ESQUISTOSSOMOSE MANSÔNICA
}

\author{
R. Dietze e A. Prata
}

\begin{abstract}
Em uma área endêmica onde havia interrupção da transmissão, foram tratados 164 esquistossomóticos com a associação oxamniquine e praziquantel em doses reduzidas. Cada individuo tomou de uma só vez uma cápsula de $250 \mathrm{mg}$ de oxamniquine e um comprimido de $300 \mathrm{mg}$ de praziquantel, ingeridos na nossa presença. As doses de oxamniquine variaram de 3,5 a $16,6 \mathrm{mg} / \mathrm{kg}$ de peso corporal e as de praziquantel de 4,2 a $20 \mathrm{mg}$.

$O$ controle de cura constou de oito exames parasitológicos de fezes de cada paciente, pelo método de Kato-Katz, num periodo de 6 meses. Os indices de cura variaram de $30 \%$ a $56,6 \%$. A percentagem total de cura foi de $39,6 \%$. A tolerância à associação foi boa. Nossos resultados mostraram baixo percentual de cura e aparente ausência de sinergismo da associação oxamniquine e praziquantel em doses reduzidas no tratamento da esquistossomose mansônica no Brasil.
\end{abstract}

Palavras chaves: Esquistossomose. Tratamento. Associação oxamniquine e praziquantel.

Hả tempos fomos informados (N Katz: comunicação pessoal) que a associação oxamniquine e praziquantel possuia ação sinérgica no tratamento da esquistossomose mansoni. Shaw e Brammer ${ }^{6}$ confirmaram o fato em camundongos e sugeriram que baixas doses de ambos os medicamentos, além da redução no custo e efeitos colaterais, poderiam ser eficazes no tratamento dessa helmintose no homem. Pugh $\mathrm{e}$ Teesdale $^{5}$ trataram 123 escolares portadores de esquistossomose mansônica e hematóbica com oxamniquine em baixas doses, mostrando ser essa associação eficaz na redução da carga parasitária e praticamente desprovida de efeitos colaterais se comparados aos de cada droga separadamente. Campos e col $^{1}$ não evidenciaram a eficácia da associação em camundongos tratados no periodo pré-patente da infecção por Schistosoma mansoni, mas no tratamento de 35 pacientes adultos portadores de esquistossomose, Campos e cols ${ }^{2}$ obtiveram indice de cura de $96,6 \%$.

Este trabalho teve por objetivo verificar a eficácia dessa associação no tratamento da esquistossomose mansônica em uma área endèmica em interrupção da transmissão.

\section{MATERIAL E MÉTODOS}

O estudo foi realizado em Caatinga do Moura (BA), como parte de um projeto longitudinal de controle da esquistossomose que vem sendo desen-

Núcleo de Medicina Tropical e Nutrição da Universidade de Brasilia.

Trabalho realizado com o auxilio do Convennio FUB/Walter Reed, Cadastro n. 08280132.

Recebido para publicaçào em 24/9/85. volvido naquela região desde 1982 . O grupo de pacientes tratados constou de 164 esquistossomóticos, diagnosticados através de um exame quantitativo de fezes pelo método de $\mathrm{Kato}-\mathrm{Katz}^{4}$. Todos os pacientes haviam sido tratados em 1983 com oxamniquine sem contudo apresentarem negativação do exame de fezes.

A idade variou de 4 a 70 anos com média de 13,8 e mediana de 11 anos. Em relação ao sexo, $83(50,6 \%)$ eram do sexo masculino e $81(49,4 \%)$ do sexo feminino. $O$ número de ovos por grama de fezes variou de 24 a 4.704 ovos com média de 164,2 e mediana de $72 \mathrm{ovos} / \mathrm{g}$ de fezes. Todos os pacientes foram tratados, independentemente do peso, com uma cápsula de oxamniquine $(250 \mathrm{mg}$ ) e um comprimido de praziquantel $(300 \mathrm{mg})$, dados simultaneamente, em dose única, ingeridos na nossa presença. A oxamniquine foi obtida do Ministério da Saúde (SUCAM) e o praziquantel diretamente da Merck. Os pacientes foram divididos em três grupos segundo a idade de 4 a 9 anos, 10 a 15 anos e maiores de 15 anos, com 64,70 e 30 individuos, respectivamente em cada grupo. No primeiro grupo as doses de oxamniquine por quilo de peso corporal variaram de 6,25 a $16,6 \mathrm{mg}$ e as de praziquantel de 8,3 a $20 \mathrm{mg}$. No segundo grupo as doses de oxamniquine variaram de 4,9 a $13,1 \mathrm{mg}$ e as de praziquantel de 5,8 a $15,7 \mathrm{mg}$. No terceiro grupo foram respectivamente de 3,5 a $6,7 \mathrm{mg}$ e de 4,2 a $8,1 \mathrm{mg}$ por quilo de peso corporal. As médias das doses de oxamniquine e praziquantel por $\mathrm{kg}$ de peso no primeiro, segundo e terceiro grupo foram respectivamente de 10,9 e 12,6 de 7,6 e 9,2 e de 4,5 e $5,4 \mathrm{mg}$. As medianas das doses de oxamniquine e praziquantel foram respectivamente de 10,8 e 12,5 no grupo de 4 a 9 anos, de 7,3 e 8,8 no grupo de 10 a 15 anos e de 4,3 e 
Dietze R, Prata A.Baixa eficácia da associaçăo oxamniquine e praziquantel na cura da esquistossomose mansônica. Revista da Sociedade Brasileira de Medicina Tropical 19: 247-249, Out-Dez, 1986.

5,2 no grupo com idade superior a 15 anos. Cada paciente ou responsável pelo mesmo foi informado que deveria voltar à presença do médico em caso de manifestações de intolerância ou efeitos colaterais.

$O$ controle de cura constituiu em dois exames de fezes, a cada 45 dias pelo método de Kato-Katz, por um periodo de seis meses. No total foram realizados oito exames de fezes de cada paciente. Um único exame de fezes positivo para ovos de $S$. mansoni no seguimento, incluia o paciente no grupo não curado.

A análise estatística dos resultados foi feita através do teste de Fisher a um nivel de significancia de 0,05 .

\section{RESULTADOS}

No controle de cura $131(79,9 \%)$ pacientes realizaram os oito exames programados, $12(7,3 \%)$ fizeram sete, $19(11,6 \%)$ fizeram seis e $2(1,2 \%)$ fizeram somente cinco exames de fezes.

A percentagem total de cura foi de $39,6 \%$. A possibilidade de reinfecção pode ser praticamente afastada, devido ao uso periódico de moluscicida (Bayluscide) na área. Essa medida além de baixar a densidade dos caramujos, baixou a zero tanto os indices de infecção dos caramujos quanto os índices de infecção de camundongos sentinelas usados na avaliação da transmissão na área ${ }^{3}$.

De acordo com as doses dos medicamentos ficaram curados $22(48,8 \%)$ pacientes entre os 45 que tomaram oxamniquine nas doses de 3,5 a $5,9 \mathrm{mg}$ por quilo de peso e praziquantel nas doses de 4,2 a $7,1 \mathrm{mg}$. Entre os 73 que usaram os medicamentos respectivamente, nas doses de 6 a $10 \mathrm{mg}$ e 7,2 a 12,1 houve negativação dos exames de fezes para ovos de $S$. mansoni em $22(30,1 \%)$. Nos 46 medicados com 10,1 a $16,6 \mathrm{mg}$ por $\mathrm{kg}$ de peso com oxamniquine e 12 a $20 \mathrm{mg}$ de praziquantel, a cura ocorreu em $22(47,8 \%)$. Considerando agora os indices de cura nos respectivos grupos etários veremos que $27(42,1 \%)$ pacientes entre 4 e 9 anos pararam de eliminar ovos de $S$. mansoni após o tratamento. $\mathrm{Na}$ faixa etária de 10 a 15 anos ocorreu cura em $21(30 \%$ ) individuos e no grupo com idade acima de 15 anos em 17(56,6\%) (Tabela 1). Considerando as doses dos medicamentos em relação aos grupos etários de 4 a 9,10 a 15 e acima de 15 anos (Tabela 1) vemos que as percentagens de cura para cada um deles, passou com o aumento da dose de $30,5 \%$ para $53,5 \%$ de $26,6 \%$ para $32,5 \%$ e de 60 para $53,3 \%$.

Nenhum paciente voltou à presença do médico para referir queixas; assim a tolerância foi interpretada como boa e as possiveis reações não justificaram a volta dos pacientes. Aqueles que continuaram com exame de fezes positivos, serão submetidos melhor a tratamentos seletivos periódicos dentro do programa de controle da esquistossomose.

\section{COMENTÁRIOS}

Nossos resultados mostraram baixo percentual de cura e aparente ausência de sinergismo da associação oxamniquine e praziquantel em doses reduzidas no tratamento da esquistossomose mansônica.

Observamos também que, à exceção do grupo com idade entre 4 a 9 anos, não ocorreu diferença estatisticamente significativa nos indices de cura com o aumento das doses dos medicamentos (Tabela 1). A esse respeito convém ressaltar que alguns pacientes receberam mais da metade das doses de oxamniquine e cerca da metade das doses de praziquantel habitualmente usadas em nosso meio no tratamento da esquistossomose (Tabela 1). Essas doses tornam a associacào mais cara do que a utilização da oxamniquine separadamente, se considerarmos os preços atuais de mercado, o que poderia ser um fator limitante do uso dessa associação em grande escala. Nossos dados diferem daqueles obtidos por Pugh e Teesdale ${ }^{5}$ e por

Tabela 1 - Eficácia da associação oxamniquine e praziquantel no tratamento da esquistossomose mansoni nos respectivos grupos etários.

\begin{tabular}{|c|c|c|c|c|c|c|}
\hline \multirow[b]{2}{*}{$\begin{array}{l}\text { Dose } \mathrm{mg} / \mathrm{kg} \\
\text { Pacientes }\end{array}$} & \multicolumn{2}{|c|}{4 a 9 anos } & \multicolumn{2}{|c|}{10 a 15 anos } & \multicolumn{2}{|c|}{$>15$ anos } \\
\hline & $\begin{array}{l}O X 6,25 \text { a } 10,9 \\
P Z 8,3 \text { a } 13\end{array}$ & $\begin{array}{l}11 \text { a } 16,6 \\
13,4 \text { a } 20\end{array}$ & $\begin{array}{l}4,9 \text { a } 6,9 \\
5,8 \text { a } 8,3\end{array}$ & 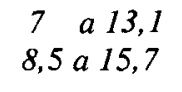 & $\begin{array}{l}3,5 \text { a } 4,3 \\
4,2 \text { a } 5,2\end{array}$ & $\begin{array}{l}4,4 \text { a } 6,7 \\
5,3 \text { a } 8,1\end{array}$ \\
\hline $\begin{array}{l}\text { Tratados } \\
\text { Curados }\end{array}$ & $\begin{array}{c}36 \\
11(30,5 \%)\end{array}$ & $\begin{array}{c}28 \\
16(53,5 \%)\end{array}$ & $\begin{array}{c}30 \\
8(26,6 \%)\end{array}$ & $\begin{array}{c}40 \\
13(32,5 \%)\end{array}$ & $\begin{array}{c}15 \\
9(60 \%)\end{array}$ & $\begin{array}{c}15 \\
8(53 \%)\end{array}$ \\
\hline $\begin{array}{l}\mathrm{OX}=\text { Oxamniquine } \\
\mathrm{PZ}=\text { Praziquantel }\end{array}$ & $\begin{array}{l}\text { este de Fisher } \\
\begin{array}{l}x=0,05 \\
4-9 \text { anos }-0,0298 \\
0-15 \text { anos }-0,3982 \\
>15 \text { anos }-0,05\end{array}\end{array}$ & & & & & \\
\hline
\end{tabular}


Dietze R, Prata A. Baixa eficácia da associação oxamniquine e praziquantel na cura da esquistossomose mansônica. Revista da Sociedade Brasileira de Medicina Tropical 19: 247-249, Out-Dez, 1986.

Campos e cols ${ }^{2}$, porém as metodologias utilizadas não são comparáveis entre si. No primeiro caso os autores se referem à redução do número de ovos e não à negativação do exame de fezes após o tratamento e Campos e cols ${ }^{2}$ trataram somente pacientes adultos das áreas endêmicas. Não existe em ambos os trabalhos qualquer referência sobre tratamentos anteriores dos pacientes. Sobre este aspecto convém ressaltar que nossos pacientes já haviam feito uso de medicação especifica anteriormente sem sucesso no que se refere à cura da parasitose. Este fato talvez possa ter influído nos baixos índices de cura por nós obtidos. Seria interessante observar se percentuais semelhantes de cura seriam obtidos em populações sem tratamento prévio. De qualquer forma não pudemos observar nenhuma vantagem na associação oxamniquine e praziquantel, mesmo em doses reduzidas, no tratamento da esquistossomose mansônica.

\section{SUMMARY}

In an endemic area where transmission of schistosomiasis has been interrupted 164 infected patients were treated with oxamniquine and praziquantel in reduced doses. Each individual took, under supervision, one $250 \mathrm{mg}$ capsule of oxamniquine and one tablet of $300 \mathrm{mg}$ of praziquantel. The doses of oxamniquine varied from 3.5 to $16.6 \mathrm{mg} / \mathrm{kilo}$ body weight and that of praziquantel from 4.2 to $20 \mathrm{mg}$.

The control of cure was eight faecal examination for eggs by the Kato-Katz method carried out over a period of six months. The indices of cure varied from 30 to $57 \%$. The total percentage of cure was $40 \%$. Tolerance was good. Our results show a low cure rate and an apparent lack of synergism of the association of oxamniquine and praziquantel in reduced doses in the treatment of Schistosoma mansoni in Brazil.

Key words: Schistosomiasis. Treatment. Association of oxamniquine and praziquantel.

\section{REFERÊNCIAS BIBLIOGRÁFICAS}

1. Campos R, Pinto PLS. Moreira AAB, Sant'Ana EJ, Padilha LAA, Levai EV, Catalano CM. Terapêutica experimental da esquistossomose mansônica em periodo pré-patente com a associação oxamniquine e praziquantel. In: Resumos do XXI Congresso da Sociedade Brasileira de Medicina Tropical. São Paulo, p. 50-51. 1985.

2. Campos R, Pinto PLS, Sant'Ana EJ, Moreira AAB, Amato Neto V, Padilha LAA, Levai EV, Catalano CM. $A$ associação de oxamniquine e praziquantel no tratamento da esquistossomose mansônica. In: Resumos do XXI Congresso da Sociedade Brasileira de Medicina Tropical. São Paulo, p. 51, 1985.

3. Dietze $R$. Controle da esquistossomose através de medidas integradas em uma área hiperendêmica. Tese de Mestrado. Fundação Universidade de Brasilia, DF, 1983.

4. Katz N, Chaves A, Pellegrino J. A simple device for quantitative stool thick-smear technique in schistosomiasis mansoni. Revista do Instituto de Medicina Tropical de São Paulo 14:397-402, 1972.

5. Pugh RN, Teesdale CH. Synergy of concurrent low dose oxamniquine and praziquantel in schistosomiasis. British Medical Journal 287:877-878, 1983.

6. Shaw JR, Brammer KW. The treatment of experimental schistosomiasis with a combination of oxamniquine and praziquantel. Transactions of the Royal Society of Tropical Medicine and Hygiene 77:39-49, 1983. 